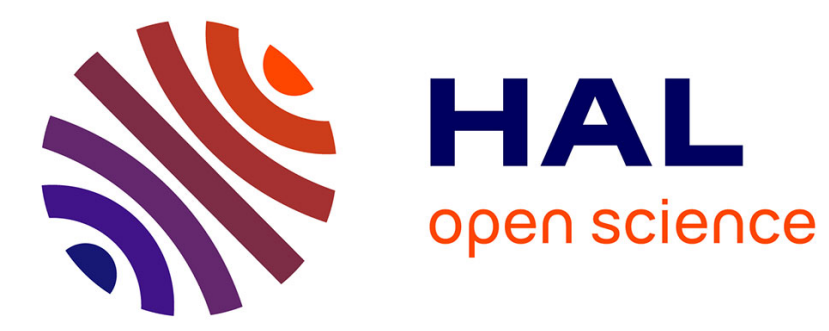

\title{
Parsimonious Path Openings and Closings
}

Vincent Morard, Petr Dokládal, Etienne Decencière

\section{To cite this version:}

Vincent Morard, Petr Dokládal, Etienne Decencière. Parsimonious Path Openings and Closings. IEEE Transactions on Image Processing, 2014, 23 (4), pp.1543 - 1555. 10.1109/TIP.2014.2303647 . hal-01082831

\section{HAL Id: hal-01082831 \\ https: / hal-mines-paristech.archives-ouvertes.fr/hal-01082831}

Submitted on 14 Nov 2014

HAL is a multi-disciplinary open access archive for the deposit and dissemination of scientific research documents, whether they are published or not. The documents may come from teaching and research institutions in France or abroad, or from public or private research centers.
L'archive ouverte pluridisciplinaire HAL, est destinée au dépôt et à la diffusion de documents scientifiques de niveau recherche, publiés ou non, émanant des établissements d'enseignement et de recherche français ou étrangers, des laboratoires publics ou privés. 


\title{
Parsimonious path openings and closings
}

\author{
Vincent Morard, Petr Dokládal and Etienne Decencière
}

\begin{abstract}
Path openings and closings are morphological tools used to preserve long, thin and tortuous structures in gray level images. They explore all paths from a defined class, and filter them with a length criterion. However, most paths are redundant, making the process generally slow.

Parsimonious path openings and closings are introduced in this paper to solve this problem. These operators only consider a subset of the paths considered by classical path openings, thus achieving a substantial speed-up, while obtaining similar results. Moreover, a recently introduced one dimensional (1-D) opening algorithm is applied along each selected path. Its complexity is linear with respect to the number of pixels, independent of the size of the opening. Furthermore, it is fast for any input data accuracy (integer or floating point) and works in stream.

Parsimonious path openings are also extended to incomplete paths, i.e. paths containing gaps. Noise-corrupted paths can thus be processed with the same approach and complexity.

These parsimonious operators achieve a several orders of magnitude speed-up. Examples are shown for incomplete path openings, where computing times are brought from minutes to tens of milliseconds, while obtaining similar results.
\end{abstract}

Index Terms-Path operators, curvilinear structures, mathematical morphology, complete and incomplete paths.

\section{INTRODUCTION}

Thin structures extraction is a non-trivial task in image processing. It requires adapted tools, used in a great range of applications, from the biomedical field to the industrial domain. Blood vessels extraction from eye fundus images [1], [2], microglia tree-like form in confocal microscope images [3], guide-wire segmentation in X-ray fluoroscopy [4], road detection from remote sensing images [5] or automated cracks detection from metallic pieces for non-destructive testing [6], [7] are some examples.

In the literature, the typical approach to enhance thin structures is to compute the supremum of openings with linear structuring elements (SE) in many orientations [8], [9]. The same strategy can be used with a bank of directional Gabor filters or difference of Gaussians filters [10]. However, tortuous structures are difficult to detect with this kind of approach. Using adaptive mathematical morphology methods improves the detection. Tankyevych et al. [11] introduced hessian based filters to detect curvilinear lines. In [7], the SE are able to adapt their shapes to enhance very thin cracks of any tortuosity. Area openings, introduced by Vincent [12], are considered as the first attribute openings, later generalized by Breen and Jones to obtain attribute thinnings [13]. Indeed, using non-increasing criteria to build attribute thinnings yields interesting filters to detect thin structures. For instance, the inertia of the connected components, weighted by their area, gives an interesting shape descriptor for elongated structures [14], [15]. More recently, thinnings based on geodesic attributes have been shown to efficiently characterize structures according to their length, tortuosity or elongation [6], [16]. Finally, the so-called path openings (PO) [17]-[19] use underlying directed acyclic graph to measure the path length.

All these methods have the same drawback: their lack of robustness with respect to noise. Indeed, thin elongated structures we are looking for can be easily corrupted by noise, resulting in disconnected paths. Heijmans et al. [19] have proposed incomplete path openings, able to deal with gaps in paths. Later, Talbot and Appleton [20] proposed an efficient algorithm to compute both complete and incomplete path openings. It has logarithmic complexity w.r.t. the length of the paths and linear w.r.t. the width of the gaps, resulting in long computation timings, unsuitable for time-critical applications. A recent work by Cokelaer et al. [21] presents a more efficient, robust to noise version of path operators, unfortunately, computing time is still not compatible with high-throughput computing.

A good robustness to noise also offers the semi-local or global approach proposed in Rouchdy and Cohen [3] or Bismuth et al. [4]. Both exploit the spatial density of locally shortest paths designed to converge to thin, curvilinear image structures to enhance. Based on this ideas, several techniques can be used to enhance or detect structures: voting [3] and voting, pruning or cost minimization in the polygonal path image [4].

The approach developed in this paper is motivated by the need to detect thin, long, tortuous and possibly noisy structures in a computationally demanding framework. The methods from the state of the art that fulfill the best these requirements are indeed path openings with complete or incomplete paths [20]. Here, we propose the Parsimonious path opening (PPO) a new fast operator for detecting the same set of structures, using complete or incomplete paths. PPO only explore a relevant subset of all paths in the image, to reduce the computation time by several orders of magnitude. Hence, the results of PPO are not exactly the same as classical path openings but they are fast, accurate and robust to noise.

This paper is organized as follows. We first recall the theory of classical path openings (Sec. II). Then, we describe the extraction of the relevant subset of paths in the image (Sec. III), the filtering strategies available (Sec. IV), the practical considerations (Sec. V) and the operator accuracy (Sec. VI). Finally, we present some results through an application: the detection of cracks from road pavement images. We also study the algorithmic complexity and we propose a timing comparison with classical path openings (Sec. VII).

\section{BASIC NOTIONS ON PATH OPENINGS}

Path openings [18], [19] were introduced to offer a higher flexibility compared to the supremum of linear openings. We briefly recall here their definition and characteristics. 


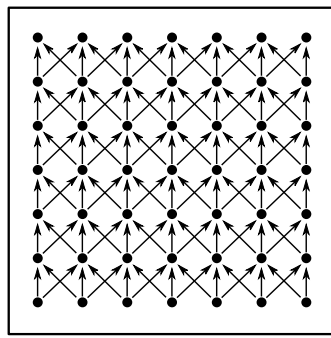

(a)

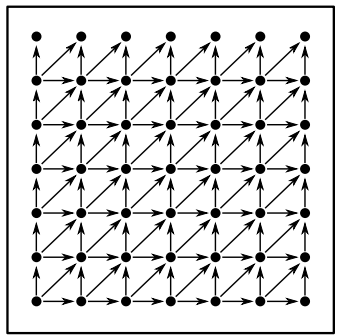

(b)

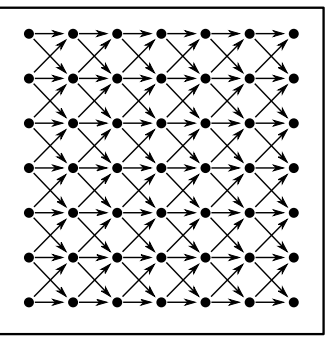

(c)

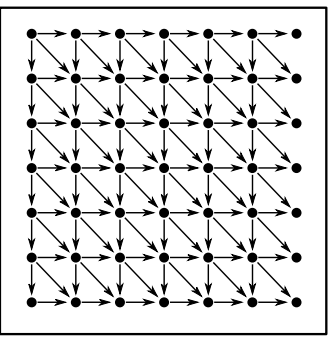

(d)

Fig. 1. Example of commonly used graphs: (a) paths having an orientation from south to north (S-N). (b) SW-NE paths, (c) W-E paths and (d) NW-SE paths

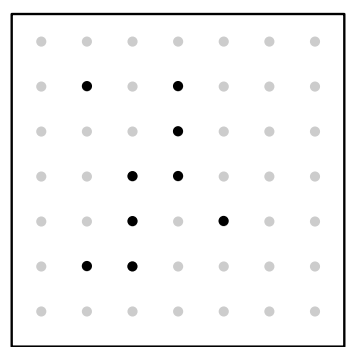

(a)

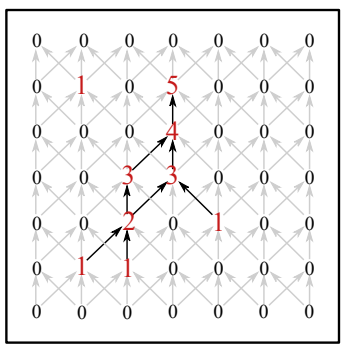

(b)

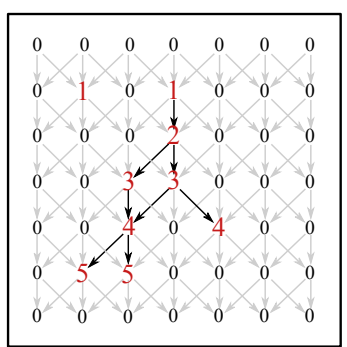

(c)

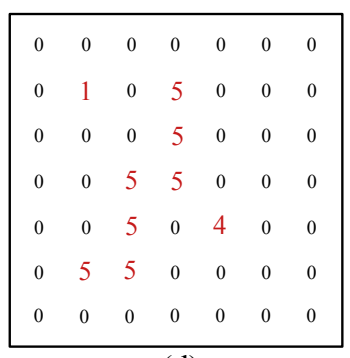

(d)

Fig. 2. Path openings computation: (a) input binary image, (b) upstream distance map $\lambda^{+}$, (c) downstream distance map $\lambda^{-}$, and (d) maximal path length map $\lambda$.

\section{A. Connectivity graph and maximal paths}

A two-dimensional binary image $X$ can be described as a subset of a rectangular sub-domain $D$ of $Z^{2}$. We equip $D$ with a directed acyclic graph $G: D \rightarrow \mathscr{P}(D)$, where $\mathscr{P}(D)$ is the power set of $D$. For any two points $x$ and $y$ of $D$, we say that $x$ is linked to $y$ on $G$ if, and only if, $y \in G(x) . G^{-}$is the inverse of $G$, defined by $G^{-}: D \rightarrow \mathscr{P}(D)$ and for all $x$ in $D, y \in G^{-}(x)$ (i.e. $x$ is linked to $y$ on $G^{-}$) if, and only if, $x \in G(y)$ (i.e. $y$ is linked to $x$ on $G$ ). $G_{X}$ is the subgraph of $G$ obtained when the graph is restricted to $X$. $G$.

Fig. 1 illustrates some classical graphs used in practice for

Let us introduce now the definition of a path on $G_{X}$ :

\section{Definition 1}

A sequence $\pi=\left(x_{1}, x_{2}, \ldots, x_{n}\right), n \in \mathbb{N}$, of points is a path of $G_{X}$ if, and only if, $\forall i \in \mathbb{N}, 1 \leq i \leq n-1, x_{i+1} \in G_{X}\left(x_{i}\right)$ ( $x_{i}$ is linked to $x_{i+1}$ on $G_{X}$ ). The path length is equal to $n$. Points $x_{1}$ and $x_{n}$ are its starting and end points. The set of all paths of $G_{X}$ is denoted $\Pi_{G_{X}}$.

On each point $x$ of $D$ we define $\lambda_{G_{X}}(x)$ (or $\lambda(x)$, when there is no ambiguity) as the maximal length of all the paths of $\Pi_{G_{X}}$ going through $x$. If $x$ does not belong to $X$, then $\lambda(x)$ is equal to zero. Given that the considered graphs are finite, and without loops, the values of $\lambda$ are finite.

\section{B. Binary path opening}

Map $\lambda$ can be efficiently computed using a scan of $G_{X}$, followed by a scan of $G_{X}^{-}$: let $\lambda^{+}$(reps. $\lambda^{-}$) be the map which gives, for each point $x$ of $D$, the maximal length of the paths of $\Pi_{G_{X}}$ (resp. $\Pi_{G_{X}^{-}}$) ending at $x . \lambda^{+}$and $\lambda^{-}$are efficiently computed thanks to the following equations:

$$
\begin{aligned}
& \lambda^{+}(x)=\max _{y \in G_{X}(x)} \lambda^{+}(y)+1, \\
& \lambda^{-}(x)=\max _{y \in G_{X}^{-}(x)} \lambda^{-}(y)+1 .
\end{aligned}
$$

An example of $\lambda^{+}$and $\lambda^{-}$is shown in Fig. 2 (b and c). Finally, $\lambda$ is simply computed as follows, for all $x \in D$ :

$$
\lambda(x)=\lambda^{+}(x)+\lambda^{-}(x)-1 .
$$

For a given pixel $x, \lambda(x)$ gives the length of the longest paths going through it. If we remove from $X$ all the points where $\lambda$ is smaller than a given constant $L$, we obtain an operator which is idempotent, increasing and anti-extensive (see Heijmans et al. [19] for the details), therefore, it is an opening, called a path opening (PO) of size $L$, and written $\Gamma_{L}^{\mathrm{PO}}(X)$ :

$$
\Gamma_{L}^{\mathrm{PO}}(X)=\left\{x \in X \mid \lambda_{G_{X}}(x) \geq L\right\} .
$$

Such an opening, when based on any of the graphs illustrated in Fig. 1, is by design not rotation invariant, an often welcome property. In order to improve on this aspect, one can use the fact that the supremum of openings is still an opening [22]: four openings are in practice computed, based on the four graphs depicted in Fig. 1, and their supremum is computed.

In the following $\Gamma_{L}^{\mathrm{PO}}$ will denote the path opening of size $L$ based on this supremum.

\section{Gray level path openings}

Let $f: D \rightarrow V$ be a gray level image, where $V$ is a finite subset of $\mathbb{R}$, such as $\{0,1, \ldots, 255\}$. Finally, $\infty_{V}$ and $-\infty_{V}$ respectively denote the maximal and minimal values of $V$. 
Let $X^{h}=\{x \mid f(x) \geq h\}$ be the upper level set obtained by thresholding $f$ at level $h$. Given that the binary opening $\Gamma_{L}^{\mathrm{PO}}$ is increasing, it commutes with thresholding. Thence, the extension to gray level images is direct:

$$
\forall x \in D, \gamma_{L}^{\mathrm{PO}}(f)(x)=\vee\left\{h \in V \mid x \in \Gamma_{L}^{\mathrm{PO}}\left(X^{h}(f)\right)\right\} .
$$

Using equation 5 to compute gray level path openings is easy, but it is never used in practice because it would be much too slow. In [23], an efficient update of $\lambda^{+}$and $\lambda^{-}$ is proposed, which achieves a large speed up factor. This update is improved in Luengo Hendriks [24] to be able to easily work with $n-D$ images. The same paper [24] also proposes a constraint on the connectivity to prevent paths from zigzagging and overestimating the length of diagonally oriented segments. Later, Cokelaer et al [21] use a different way of making the path opening less sensitive to noise with $n-D$ images. These three papers bring notable improvements on path opening timings, but the algorithms are still too slow for many applications. Parsimonious path operators, presented in the following sections, address this issue.

In what follows, we present and illustrate this work with the extraction of bright structures in an image, with no loss of generality. Path closings (resp. parsimonious path closings) are computed using path openings (resp. parsimonious path openings) on the inverted image.

\section{PARSIMONIOUS SET OF PATHS}

The principal idea behind the notion of parsimonious paths is to work with a restricted set of paths instead of exploring all of them. In fact, the set of paths will be so sparse, that most points in the image will not be crossed by any of them.

In the original definition of path openings, the number of paths grows exponentially with the size of the image. However, only few out of these paths bring relevant information. In the following, we deal with the problem of building a relevant subset of paths.

For the extraction of bright structures, the relevant paths have to follow the brightest structures of the image. Since this will usually leave other pixels devoided of a path, we will speak about parsimonious path openings.

Three strategies are proposed in this section to select a relevant subset of paths. The last one is a generalization of the first two. Hereafter, $D$, the definition domain or support of $f$, will be a rectangular subset of $\mathbb{Z}^{2}$, and $G$ will be a directed acyclic graph.

\section{A. Locally maximal paths}

The strategy called locally maximal paths (LMP) performs a local search for bright structures. Definition 1 relative to a path is extended as follows:

\section{Definition 2}

$$
\begin{aligned}
& \pi^{\mathrm{LMP}}=\left(x_{1}, \ldots x_{n}\right) \text { is a locally maximal path if, and only } \\
& \text { if, the starting point of the path belongs to a boundary of } D \\
& \text { and if, } \forall x_{i} \in \pi^{\mathrm{LMP}}, 0 \leq i \leq n \text {, we have: } \\
& \qquad x_{i+1} \in \underset{x_{j} \in G\left(x_{i}\right)}{\operatorname{argmax}}\left\{f\left(x_{j}\right)\right\} .
\end{aligned}
$$

Equation 6 is used to iteratively construct a path from a starting point. The path ends when there is no successor to $x_{n}$. We note that several successors of a pixel $x_{i}$ may have the same gray-scale value. In that case, the principal orientation is preserved by selecting the central pixel defined by the graph.

Pixels from the boundary of $D$ are used as starting points for each selected graph. Thus, we define $\Pi_{f}^{\mathrm{LMP}}$ :

\section{Definition 3 \\ The set $\Pi_{f}^{\mathrm{LMP}}=\left\{\pi_{1}^{\mathrm{LMP}}, \ldots, \pi_{p}^{\mathrm{LMP}}\right\}$, is the set of locally maximal paths of $f$.}

Fig. 4(b) proposes an illustration of this set. Pixels that belong to at least one path of the set appear as white ; other pixels are black. The original image shows a molecule of DNA observed with an electron microscope [18] (Fig. 4(a)). We note that the number of paths in the image is very low in comparison with the number of paths considered by path openings. We also observe that most pixels are black (no path crossing them). This method is not only sparse with respect to paths, but also with respect to pixels.

With this strategy, the search for the next pixel of a path is only local and the required time is very low. However, such paths are not very robust to noise. For instance, impulsive noise can disturb and deviate a path from a thin structure. To improve noise robustness, we make a global search for the paths with a second strategy, namely globally maximal paths (GMP).

\section{B. Globally maximal paths}

To build a path, we use graph theory to search for the highest path between two pixels of the image. To explain the notion of highest path, let us see the image as a topographical surface where high (resp. low) gray-scale values correspond to high (resp. low) altitudes. A globally maximal path (GMP) is a path between two points such that the average grayscale value is the highest one among all available paths. The Dijkstra algorithm allows such search. However, given that the graph is directed and acyclic, specific algorithms can be used to provide fast algorithms. They are part of dynamic programming approaches and known as longest path algorithms [25]-[28]. The definition of a globally maximal path is given as follows:

\section{Definition 4}

$\pi^{\mathrm{GMP}}=\left(x_{1}, \ldots x_{n}\right)$ is a globally maximal path if, and only if, the starting and end points of the path belong to a boundary of $D$ and if we have:

$$
\pi^{\mathrm{GMP}} \in \underset{\pi \in \Pi_{G}}{\operatorname{argmax}}\left(\frac{1}{\operatorname{card}(\pi)} \sum_{x_{i} \in \pi} f\left(x_{i}\right)\right) .
$$

For a given point from the boundary of $D$, several globally maximal paths can be found. In practice, we select the path that preserves the principal orientation of the graph. Computing a GMP from all boundary pixels of the support $D$ and for every considered graph, we obtain $\Pi_{f}^{\mathrm{GMP}}$ : 


\section{Definition 5}

$$
\mid \begin{aligned}
& \Pi_{f}^{\mathrm{GMP}}=\left\{\pi_{1}^{\mathrm{GMP}}, \ldots, \pi_{p}^{\mathrm{GMP}}\right\}, \text { is the set of globally maximal } \\
& \text { paths of } f .
\end{aligned}
$$

This set is depicted on Fig. 4(g). We observe that the paths tend to go straight towards a bright structure, and then they follow it as far as they can. It is a global approach, robust to noise. With this strategy, the size of zones with no information (no path going through them) is larger than with locally maximal paths. We call these zones blind regions, since structures localized in these regions are not analyzed. With globally maximal paths, large and bright structures attract all paths; short and a bright structures found in their vicinity might not be seen.

GMP need more computation time than LMP and the size of blind regions is larger. Nevertheless, the robustness w.r.t noise is much higher than with LMP. Below we introduce a new general formalism that allows for intermediate strategies. We call this generalization the $\beta$-maximal paths ( $\beta \mathrm{MP})$.

\section{C. $\beta$-maximal paths}

The idea of $\beta \mathrm{MP}$ is to localize the globally maximal paths by subdividing the image support. With a given graph, say south to north (Fig. 1(a)), the image is divided into several horizontal stripes $\beta$ pixels height, as illustrated in Fig. 3.

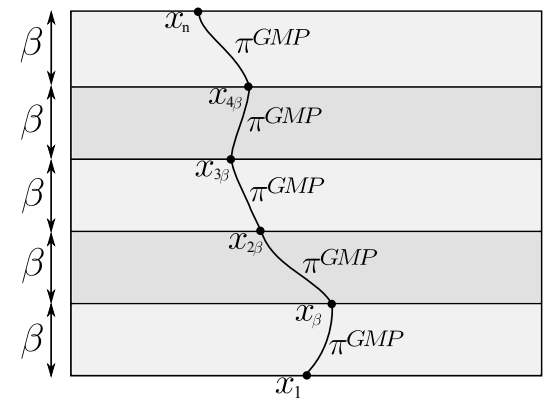

Fig. 3. Construction of $\beta$ maximal paths with the concatenation of globally maximal paths.

From a starting point $x_{1}$ localized on the bottom boundary of the support $D$, we compute $\pi^{\mathrm{GMP}}=\left(x_{1}, \ldots, x_{\beta}\right)$ on the first stripe. Then, $x_{\beta}$ is the new starting point and we iterate this process until there is no successor to $x_{n}$ with the graph $G$.

\section{Definition 6}

$\pi^{\beta \mathrm{MP}}=\left(x_{1}, \ldots, x_{n}\right)$ is a $\beta$ maximal path if, and only if, the starting point of the path belongs to a boundary of $D$ and if $\pi^{\beta M P}$ is the concatenation of globally maximal paths $\pi^{\mathrm{GMP}}$ on stripes of size $\beta$.

Computing $\beta$ MP from all boundary pixels of the support $D$ and for every graph $G$, we get $\Pi_{f}^{\beta \mathrm{MP}}$ :

\section{Definition 7}

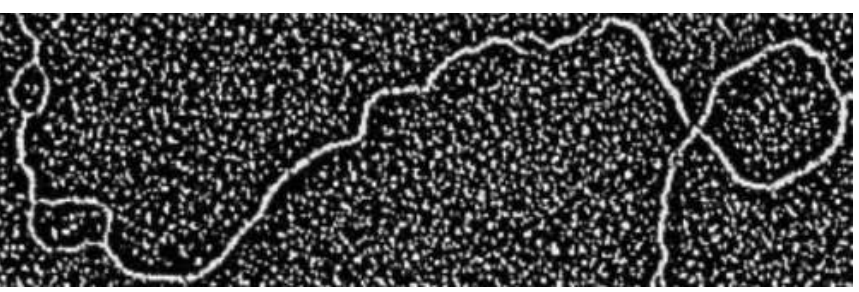

(a) Input image $(500 \times 160$ pixels $)$

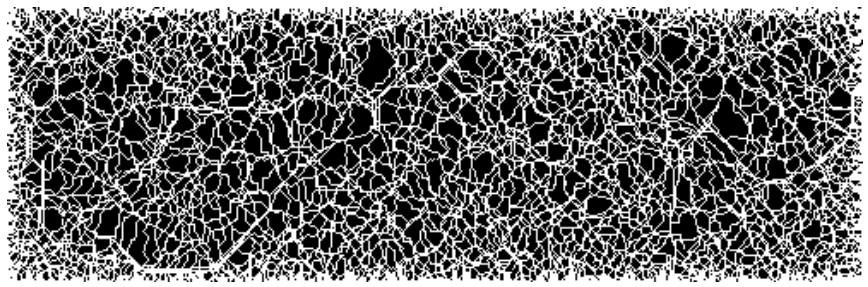

(b) $\Pi^{\mathrm{LMP}}$ : locally maximal paths $(\beta=1)$

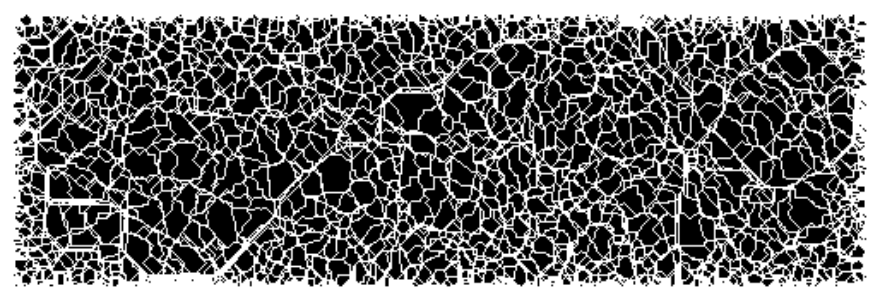

(c) $\Pi^{5 \mathrm{MP}}: \beta$ maximal paths $(\beta=5)$

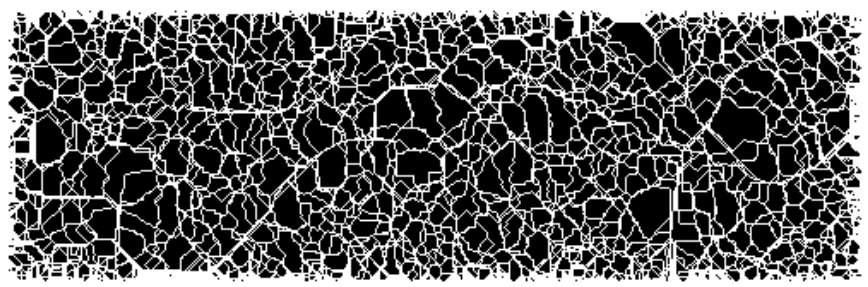

(d) $\Pi^{10 M P}: \beta$ maximal paths $(\beta=10)$

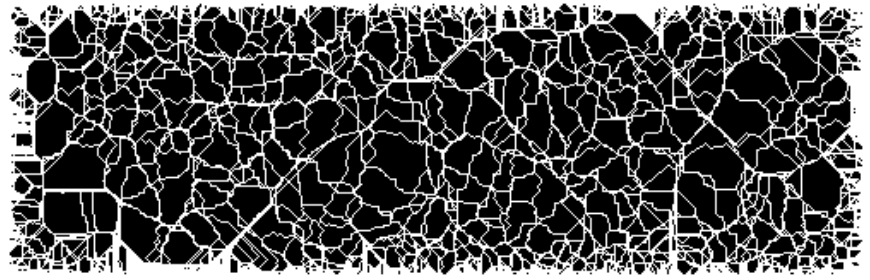

(e) $\Pi^{30 \mathrm{MP}}: \beta$ maximal paths $(\beta=30)$

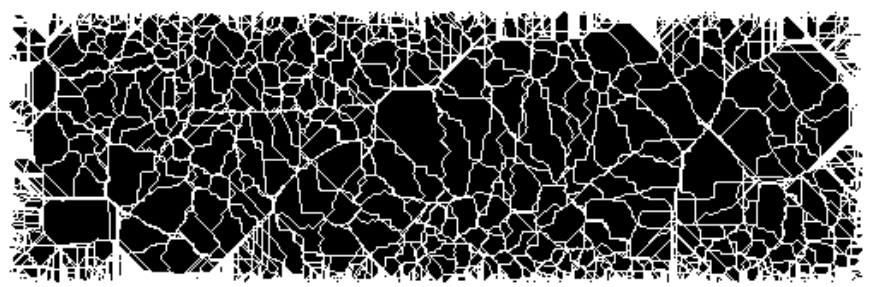

(f) $\Pi^{50 \mathrm{MP}}: \beta$ maximal paths $(\beta=50)$

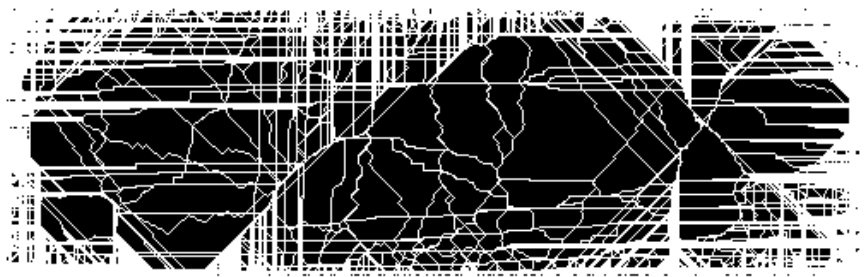

(g) $\Pi^{\mathrm{GMP}}$ : global maximal paths $(\beta=\infty)$

Fig. 4. Illustration of the sets of parsimonious paths on a given image (a). Pixels in white belong to at least one path of the set; other pixels appear in black. The graphs used are defined in Fig. 1. 


$$
\mid \begin{aligned}
& \Pi_{f}^{\beta \mathrm{MP}}=\left\{\pi_{1}^{\beta \mathrm{MP}}, \ldots, \pi_{p}^{\beta \mathrm{MP}}\right\}, \text { is the set of all the } \beta \text { maximal } \\
& \text { paths of } f .
\end{aligned}
$$

By definition, $\beta$ maximal paths generalize previous methods: LMP are obtained with $\beta=1$ and GMP with $\beta=\infty$. This method unifies the path extraction strategy and is used to compute the set of paths of Fig. 4. Thus, we control the trade-off between noise robustness and blind regions size. The choice of $\beta$ is application dependent. On a noisy image, a high value for $\beta$ is preferable. On the contrary, if the signal to noise ratio is high, a small value will reduce the size of blind regions.

Now that we have proposed a general strategy to extract parsimonious paths, below we explain how to:

- filter an image along each path;

- combine the results along each path to obtain a 2-D operator.

\section{PATHS OPERATORS}

We will see in this section how, from an operator working on single paths, we can build an operator working on the whole image.

\section{A. General strategy}

Recall that $D$ is the support of function $f$, also written $\operatorname{spt}(f)$, and $\Pi=\left\{\pi_{i}\right\}$ denotes a collection of paths of $D$. The restriction of $f$ to the path $\pi$, denoted by $f_{/ \pi}$, can be considered as a one-dimensional signal. Furthermore, recall that the set of values $V$ is a finite subset of $\mathbb{R}$.

Let $\xi f_{/ \pi}$ be the application of a 1-D operator $\xi$ to $f$ restricted to $\pi$. Using $\xi$ we obtain a result for each path $\pi_{i}$ of $\Pi$. Notice that for any $x$ belonging to the intersection of two different paths $\pi_{i}$ and $\pi_{j}$, one generally obtains $\xi f_{/ \pi_{i}}(x) \neq$ $\xi f_{/ \pi_{j}}(x)$. Hence, we need a method to produce a single value.

Let $\operatorname{spt}(\Pi)$ denote the support of $\Pi$, i.e. the set of all points of $D$ which belong to at least one path of $\Pi$. The illustrations of different sets of parsimonious paths given in Fig. 4 correspond in fact to this domain. We can extend $\xi f_{/ \pi}$ to $\operatorname{spt}(\Pi)$ by taking:

$$
\xi f(x)=\underset{\substack{\pi \ni x \\ \pi \in \Pi}}{\otimes} \xi\left(f_{/ \pi}\right)(x),
$$

where $\otimes$ is a binary operator such as $\bigvee$ or $\bigwedge$ (i.e. supremum or infimum). In practice, the choice of the binary operator will depend upon the desired properties of the resulting operator. Several examples are described in the following sections.

We now have a result on all points belonging to at least one path. However, Eq. 8 does not define $\xi f(x)$, for $x$ outside the support of $\Pi$.

The $\beta$-maximal strategy constrains the paths to go through the brightest structures of the image (the support of the paths $\operatorname{spt}(\Pi)$ ). These are usually the structures of interest. Even though one may focus only on these objects, e.g. for measuring purposes, having a result for each pixel of the image support $(\operatorname{spt}(f))$ can be useful, e.g. for filtering or preprocessing purposes.

The first and simpler strategy consists of using a constant value outside $\operatorname{spt}(\Pi)$, e.g. the minimum or maximum of $V$, or even the original $f$. Another strategy is using a morphological reconstruction under/above $f$ in order to propagate the results to the entire $D$. We will come back to this strategy in section V-D.

\section{B. Parsimonious path openings}

The first step to obtaining parsimonious path openings (PPO) is the choice of a convenient $\xi$. We naturally take the 1-D opening of size $L, \gamma_{L}$ and use $\bigvee$ in Eq. 8 to compute a value for each $x \in \operatorname{spt}(\Pi)$ :

$$
\gamma_{L}^{\Pi}(f)(x)= \begin{cases}\underset{\substack{\pi \ni x \\ \pi \in \Pi}}{\bigvee} \gamma_{L}\left(f_{/ \pi}\right)(x), & x \in \operatorname{spt}(\Pi) \\ -\infty_{V} & \text { otherwise }\end{cases}
$$

we pad $D$ outside $\operatorname{spt}(\Pi)$ by $-\infty_{V}$ to ensure the antiextensiveness of the operator.

Based on the fact that $\gamma_{L}^{\Pi}$ is built from openings using supremums, and that outside $\operatorname{spt}(\Pi)$ the result is set to the minimal value of $V$, it can be demonstrated that this operator is an opening.

Note that if $\Pi$ covers all the nodes of the graph, then $\operatorname{spt}(\Pi)=D$ and no padding is necessary.

Concerning path closings, as in the classical path opening definition by Heijmans et al. [19], they can be obtained by duality: $\varphi(f)=-\gamma(-f)$. However it should be noted that $\Pi_{f}$ and $\Pi_{-f}$ are not the same. Whereas $\Pi_{f}$ selects the bright structures $\Pi_{-f}$ selects the dark ones.

\section{Interlude: why parsimonious path openings are openings?}

The set of paths $\Pi$ is a function of the image $f$, written $\Pi_{f}$. So, what can we say about $\gamma_{L}^{\Pi_{f}}$ ? It can be shown that although anti-extensivity remains a valid property, increasingness and idempotence are lost. Therefore this more general operator is not an opening.

From a practical point of view, to avoid an unexpected behavior of serially composed operators, once $\Pi_{f}$ is defined, it should remain constant. For example, for building granulometries [29] (see Section VI later) it is necessary to use the same $\Pi$ in all stages. Similarly, when computing alternate sequential filters, it is logical to compute once $\Pi_{f}$ and $\Pi_{-f}$ and use them, respectively, in computing all subsequent openings and closings.

Hence, provided the collection of extracted paths $\Pi_{f}$ remains constant, PPO are anti-extensive, increasing and idempotent operators. Consequently, we can conclude that PPO are openings.

\section{Parsimonious Incomplete path openings (PIPO)}

In presence of noise, long, thin structures are likely to appear disconnected, implying an underestimation of their length. See for example the result of a PPO in Fig. 5. Notice that while the background texture has been filtered out, some portions of the molecule are lost as well. We propose solutions below.

Consider a signal disconnected by one "noisy" pixel (Fig. 6, top). A path opening $\gamma_{L}$ fails to detect a part of it (Fig. 6, 


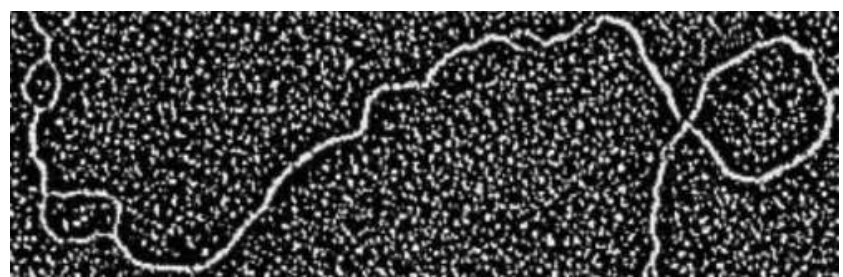

(a) Input image $(500 \times 160$ pixels $)$

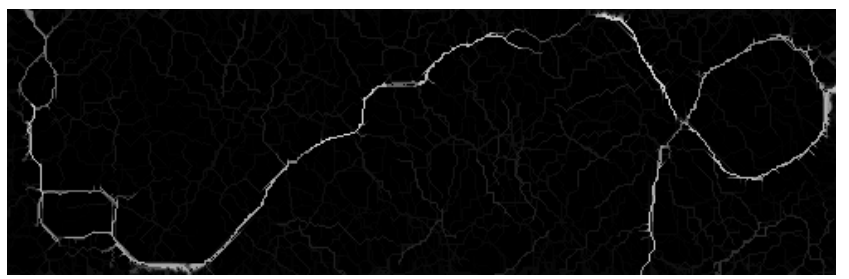

(b) PPO $\gamma_{L}^{\Pi} L=50$

Fig. 5. A molecule (a) on textured background. The PPO nicely filters background noise, but the contrast of some molecule sections is partially lost. The graphs used are defined in Fig. 1.
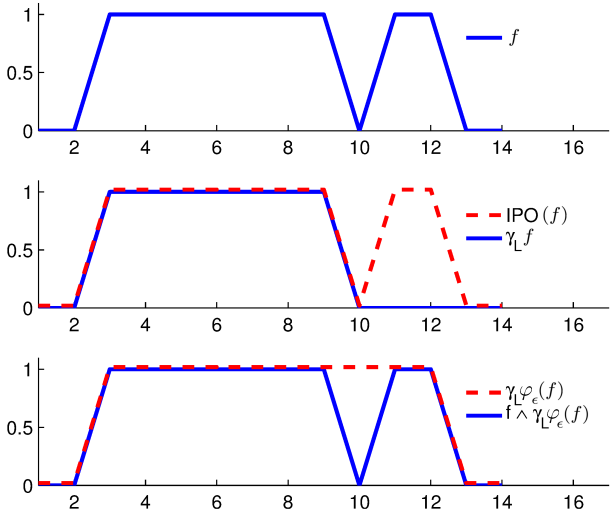

Fig. 6. Complete and incomplete parsimonious path openings. From top to bottom: input signal $f$. The opening $\gamma_{L}(f), L=5$ fails to detect a portion of the signal due to noise. A closing $\varphi_{\epsilon}(f)$ of size $\epsilon=2$, followed by the opening, $\gamma_{L} \varphi_{\epsilon}(f)$, detects correctly the entire structure. The infimum with the original $f \wedge \gamma_{L} \varphi_{\epsilon}(f)$ is used to ensure the anti-extensiveness of the result.

middle, blue). To solve this problem, two strategies have been proposed in the literature. In the following, $\epsilon>0$ is a general parameter corresponding to the robustness with respect to noise. Talbot and Appleton [20] introduced Incomplete Path Openings (IPO), tolerating gaps (Fig. 6, middle, dashed red) in the same way as rank max openings. For example, an IPO of length 50 with $\epsilon=1$ may not have less than 49 pixels, and no more than one missing pixel. Robust path operators (RPO), introduced by Cokelaer et al. [21], tolerate gaps up to some maximal allowable width. For example, a robust path [21] of length 50 with $\epsilon=1$ may contain as few as 26 pixels, with one-pixel gaps in between.

To achieve the same objective within our framework, we propose to use a small closing $\varphi_{\epsilon}$ of size $\epsilon$ to close the gap, followed by an opening of size $L$, see Fig. 6 bottom, dashed red. Therefore, $\epsilon$ is the noise tolerance parameter which behaves similarly to that of robust paths. The 2-D operator version is built by using the supremum:

$$
\zeta_{L, \epsilon}^{\Pi}(f)(x)= \begin{cases}\bigvee_{\substack{\ni \rightarrow x \\ \pi \in \Pi}} \gamma_{L} \varphi_{\epsilon}\left(f_{/ \pi}\right)(x), & x \in \operatorname{spt}(\Pi) \\ -\infty_{V} & \text { otherwise. }\end{cases}
$$

The original IPO verify the anti-extensivity of openings. To obtain an anti-extensive result from Eq. 10 one can take the infimum with the original image to obtain a parsimonious incomplete path opening $\gamma_{L, \epsilon}^{\Pi} f=f \wedge \zeta_{L, \epsilon}^{\Pi} f$ (see Fig. 6 bottom, blue).

\section{PRACTICAL CONSIDERATIONS}

The construction of PPO comprises two steps: path extraction and path processing. We give here some details on the algorithms used to speed up both steps. Next, we introduce a parsimony parameter and we allow the detection of thick structures by assigning a value to all pixels of the resulting image, by means of the morphological reconstruction.

\section{A. Path extraction: efficient algorithm}

1) Local maximal paths: The LMP strategy (Sec. III-A) extracts paths from the input image with a local search, by using the definition corresponding to Eq. 6. Thanks to the locality, the LMP extraction is extremely efficient.

On the other hand, the extraction of $\beta$ maximal paths by using the definition corresponding to Eq. 7 is slow. Below, we propose an efficient implementation using a fast, two-step algorithm.

2) Globally maximal paths: The extraction of GMP is in principle similar to the classical path opening on binary sets (Sec. II-B). This method was first introduced by Schmitt [30] to extract the longest path in a binary set, and extended later to gray level images by Vincent and Jeulin [31] to detect fracture lines in porous media or to extract correlogram tracks from noisy sonar data [28].

Starting from the input image $f$ we compute the upstream $\lambda^{+}$and downstream $\lambda^{-}$weighted distance maps with $G$ and $G^{-}$, by extending equations 1 and 2 :

$$
\begin{aligned}
\lambda^{+}(x) & =\max _{y \in G(x)} \lambda^{+}(y)+f(x), \\
\lambda^{-}(x) & =\max _{y \in G^{-}(x)} \lambda^{-}(y)+f(x) .
\end{aligned}
$$

Then, we sum these two distance maps to get, for each pixel of the image, the weighted length of the longest and brightest path going through it:

$$
\lambda(x)=\lambda^{-}(x)+\lambda^{+}(x) .
$$

Finally, GMP are obtained by using the LMP search (Eq. 6), on the map $\lambda$.

To obtain $\beta \mathrm{MP}$, we divide the image into several, $\beta$-pixel wide stripes oriented perpendicularly to the main direction of the selected graph, and we compute GMP on each of these stripes. 


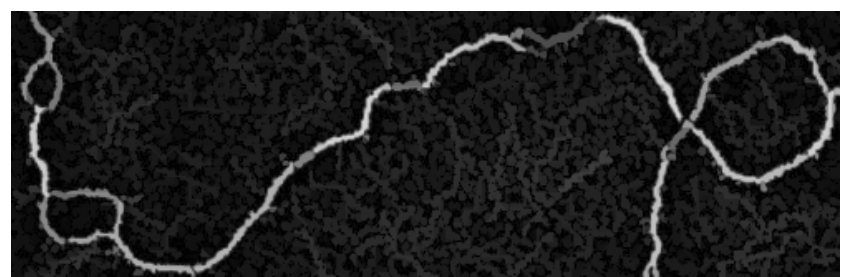

(a) PO of size $L=50$

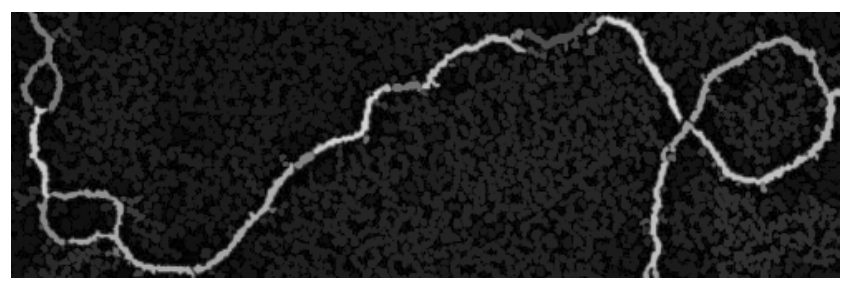

(c) PPO of size $L=50 \quad(\beta=5)$

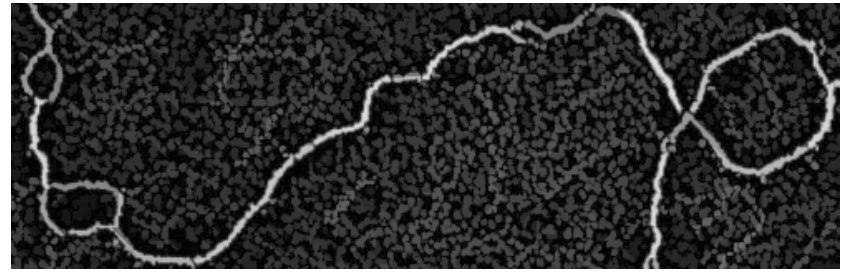

(e) RPO of size $L=50$

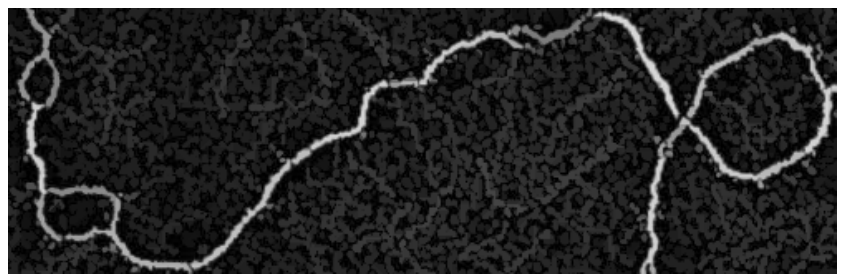

(b) IPO of size $L=50 \quad(\epsilon=2)$

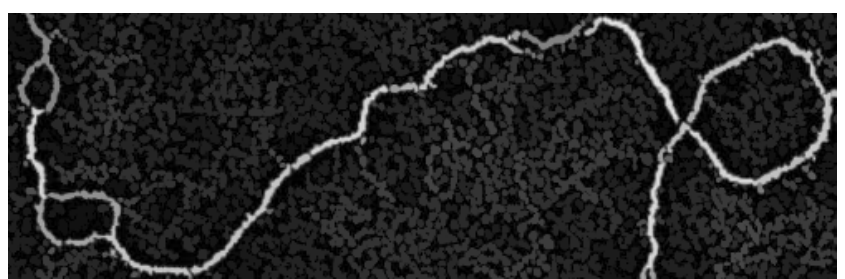

(d) PIPO of size $L=50 \quad(\epsilon=2, \beta=5)$

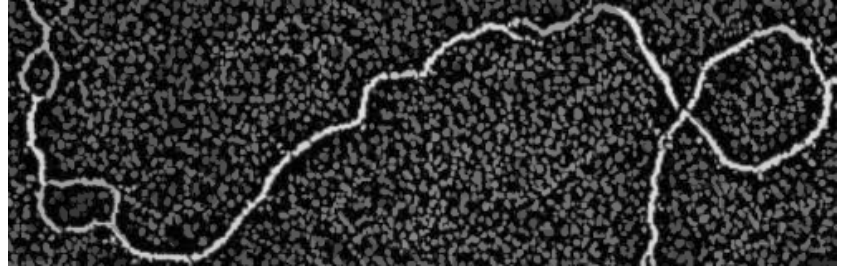

(f) RPO of size $L=50(\epsilon=2)$

Fig. 7. Comparison between path openings (first column) with parsimonious path openings with a reconstruction step (second column) for complete or incomplete paths. The graphs used are defined in Fig. 1.

To conclude, the LMP extraction and the map $\lambda$ are both computed in $\mathcal{O}(1)$ per pixel. Consequently, GMP (and $\beta \mathrm{MP}$ ) are extracted with the same $\mathcal{O}(1)$ complexity per pixel as LMP. Nonetheless, the execution time of GMP (and $\beta \mathrm{MP}$ ) is longer due to the time needed to compute $\lambda$.

\section{B. Path filtering: efficient algorithm}

The $\beta$ MP strategy individualizes each path $\pi$ of the set $\Pi^{\beta M P}$. The image alongside a path, $f_{/ \pi}$, is a $1 \mathrm{D}$ signal. Using the LMP strategy on the map $\lambda$ allows to filter while a path is being extracted. Efficient algorithms are available in the literature for 1D openings or closings that run in one scan of the signal in $\mathcal{O}(1)$ per pixel. The fastest algorithm for 1D openings is the one invented by Van Droogenbroeck and Buckley [32]. However, it uses a histogram, consequently the whole signal to be processed must be known in advance, and the pixel values are limited to 8-bits. Instead, we propose using another fast algorithm [33], [34], which offers the following additional advantages: (i) it computes the output signal progressively, each time a pixel is added to a path; (ii) it can handle the signal borders in two different ways, by padding with $-\infty_{V}$ or with $\infty_{V}$; (iii) it can handle any input data accuracy (integer or floating point) with no extra cost; (iv) the complexity is independent of the size of the opening; (v) it is fast and GPU compliant, as shown by Karas et al. [35].

In the next section, a parsimony parameter $k$ is introduced to further reduce the computation time.

\section{Parsimony parameter $k$}

Recall that all paths start at the border of the image, one path from each border point. As we see in Fig. 4, many paths rapidly converge to follow the same structures, without bringing any new information. Increasing $\beta$ further reinforces this phenomenon. The paths with close starting points are likely to be attracted by the same structures, and do not bring new information.

By reducing the number of starting points, the resulting set of paths is considerably sparser without increasing much the parsimony of the image support scan. We achieve this goal by keeping only one starting point out of $k$, the parsimony parameter of our method. The total number of paths is therefore divided by $k$, as well as the computing cost of the algorithm, while the accuracy of the PPO is only slightly reduced (see section VI for details).

\section{Morphological reconstruction}

PPO is a sparse operator and yields a thin representation of objects. Should one need a thick detection, PPO results can be reconstructed under the original image. Efficient implementations of the morphological reconstruction are available in the literature [36], [37]. Their complexity is linear with respect to the image size.

Fig. 7 illustrates the DNA molecule extraction from the noisy background using parsimonious path openings followed by a reconstruction to ease the comparison with classical path openings and with robust path openings. Figs. 7(a), 7(c) and 7 (e) compare PO, PPO and RPO with complete paths whereas Figs. 7(b), 7(d) and 7(f) illustrate the results for incomplete 
paths with a tolerance $\epsilon$ of 2 pixels. Very similar results are obtained using PPO with a significant reduction of the timings (see Sec. VII-B).

Using incomplete paths improves the detection since thin structures are reconnected. However, it also reconnects noisy pixels, preserving some structures in the image background. Thus, tuning the parameter $\epsilon$ is a trade-off between the size of the gaps to fill and the level of noise.

In the following section, the accuracy of PPO with respect to length measurements is studied.

\section{ACCURACY OF PPO}

In order to evaluate the accuracy of PPO, we will apply them to binary images containing segments of known length, and compare the measured length distribution using PPO with the theoretical one.

Size distributions are usually computed by a residual approach with a collection of increasing-size filters commonly known as granulometry, introduced by Matheron [29], [38]. Let us us consider a binary image $f$, and a set of paths $\Pi_{f}$ computed on $f$. The family of PPO $\left\{\gamma_{L}^{\Pi}\right\}_{L>1}$ is a granulometry. Note that we have dropped the $f$ index on $\Pi$, as the set of paths is computed once and for all, and then kept constant, for reasons explained in section IV-C. The corresponding size distribution is, for non-negative integers $L$ :

$$
\operatorname{SD}_{L}=\operatorname{Meas}\left(\gamma_{L}^{\Pi}-\gamma_{L-1}^{\Pi}\right) .
$$

In the present case, the measure Meas is the number of connected components of the binary image. Eq. (14) measures the length distribution.

PPO suffer from two types of error. The first one is the anisotropy of the length measurement, also present in the original PO. It comes from the discretization of the path on the $\mathbb{Z}^{2}$ grid. The second error is due to the parsimonious scan of the support. The following text analyzes the phenomena at the origin of these errors and their impact on the accuracy.

Let $x$ be a measurement, and $m$ the correct value. The relative measure error is:

$$
\text { err }=\frac{x-m}{m}
$$

A positive $e r r$ means overestimation, whereas a negative $e r r$ means underestimation.

In our case $x$ and $m$ are distributions (expressed as probability density functions or counts in case of histograms). A number of measures exist to compare probability density functions or histograms (see [39] for a review of most common distances or divergences). However, none of these suits our case for the following reasons: i) The metric behind the majority of the distances considers the probability density function as a vector in an orthogonal space. Nonetheless, the histogram bins in our case are not orthogonal. ii) A distance is always positive, which does not reflect the difference between under- and over-estimation of a measure. iii) No distance or metric is correlated to the usually used relative measure error as in eq. 15. This means that for singleton distributions

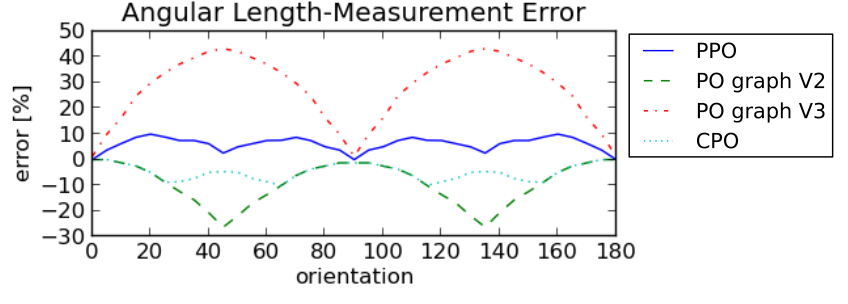

(a) thick line segment

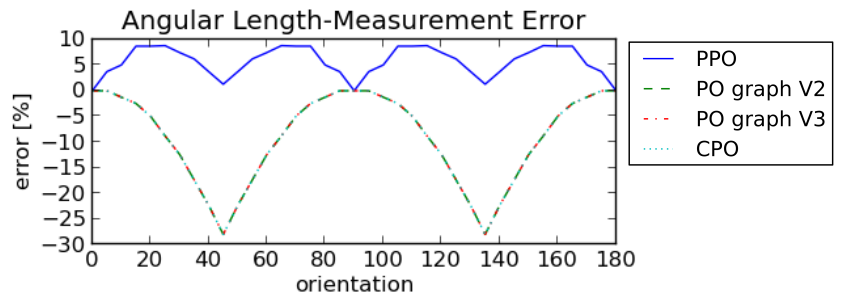

(b) thin line segment

Fig. 8. Relative error of measuring the length of a straight isolated line segment with respect to its orientation. Segment length $L=80$. (a) width $2 \mathrm{px}$, (b) thin segment. Note: For thin segments the errors of PO and CPO coincide.

(containing only one point) we would not obtain the same value as with eq. 15 .

Consequently, we define an equivalent of Eq. 15 for two histograms $X$ and $M$, with respective mean values equal to $\bar{X}$ and $\bar{M}$. We define the relative error of the measured distribution $X$ to the ground truth distribution $M$ as:

$$
\text { err }=\frac{\bar{X}-\bar{M}}{\bar{M}} .
$$

This error evaluation has the following properties: The difference $\bar{X}-\bar{M}$ is insensitive to the standard deviation of either distribution, that can be evaluated separately. It does not require the histograms to be normalized nor aligned. $X$ and $M$ can have different count sum and either $X$ and $M$ or both can be scalars. If both are scalars then eq. 16 is equivalent to eq. 15 .

The first experiment evaluates the isotropy, see Fig. 8. It reports the relative error of measuring the length of an isolated, straight segment w.r.t. its orientation. It compares PPO to path openings (PO) by Talbot and Appleton [20] and constrained path openings (CPO) by Hendriks [24]. The segment is 80 pixels long, placed in the center of the image. Since the result depends on the width, we report two cases: a two pixels thick segment, Fig. 8(a), and a thin segment, Fig. 8(b). For both, all $\mathrm{PO}, \mathrm{CPO}$ and PPO provide a correct measure for orientations multiple of $90^{\circ}$. For other orientations various phenomena occur and bring about different errors.

For diagonal orientations, PO suffer from a nonnegligible error. In thick segments, when graph V3 is used, the PO overestimate the length since the longest path zigzags around the principal orientation. When graph V2 is used, or for thin segments, the zigzagging effect does not occur. However, the PO underestimate the length since it is only evaluated by merely counting the number of pixels. 

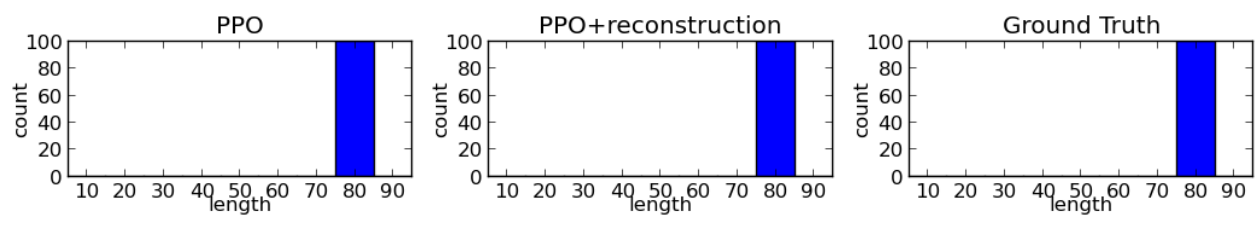

(a) orientation: $45^{\circ}$
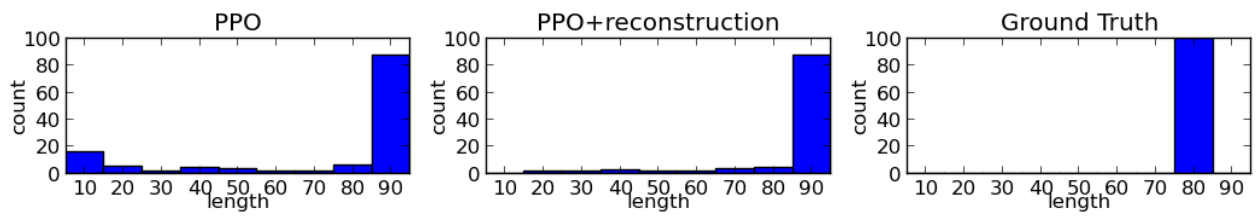

(b) orientation: $55^{\circ}$

Fig. 9. The length distribution of a population of straight, co-linear segments of constant length ( $\mathrm{L}=80$ ), oriented at $45^{\circ}$ (top), and $55^{\circ}$ (bottom), as measured with the PPO, PPO+reconstruction vs. the Ground truth (left to right)

The error due to zigzagging has already been pointed out by Hendriks and corrected by CPO [24] where the zigzagging is limited by using a more complex graph. The CPO improve the accuracy in thick objects. In thin objects, the CPO underestimate as well as the PO.

The PPO measure the length with a better accuracy than that of the two previous methods. The PPO provide a correct measure for integer multiples of $45^{\circ}$, and in all other cases, and regardless of the thickness, the error is upper-bounded by $10 \%$. This represents one of the major advances brought by PPO compared to the previous techniques.

The second experiment evaluates the error to measure the length distribution on a population of co-linear straight segments. We use a model of $N=100$, constant-length $L=80$, equally-oriented segments, randomly (uniform distribution) placed in the support without touching each other. The length distribution $M$ of the model is a Dirac impulse located at $L$ (Fig. 9, right). The length distribution $X$ measured with PPO is at left. Note that for $45^{\circ}$ (Fig. 9, top left) the measure is exact, for other orientations there is a bias towards shorter lengths (e.g. $55^{\circ}$, see the overestimated bin 10 at Fig. 9, bottom left).

The relative measure error w.r.t. the orientation (eq. 16) is given in Fig. 10 (green curve). Again, the length distribution is exact if the segments are oriented in a multiple of 45 degrees. For other orientations the two mentioned errors occur: i) the length is overestimated due to the discretization of the support, ii) a few segments have been counted in short bins. This is a direct consequence of the parsimony of the support scan. Some segments are not entirely followed by any path. For example, in Fig. 11 the segment in the middle is only partially followed by the two dotted and dashed paths. These fragments are filtered, and counted in short histogram bins.

This fragmentation can be attenuated if a morphological reconstruction is used, as proposed in section V-D. In Fig. 11, the middle segment is entirely reconstructed from its central portion used as the marker. A PPO followed by a morphological reconstruction is a connected operator [40], and yields more accurate results, see Fig. 9 middle, and Fig. 10 blue curve.

The third experiment evaluates the relative error w.r.t. the

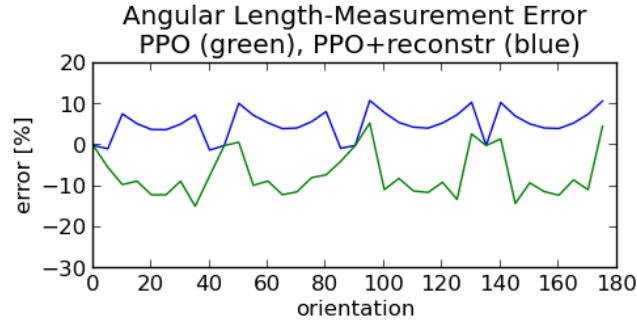

Fig. 10. The relative length-measurement error of a population of co-linear segments with respect to the orientation.

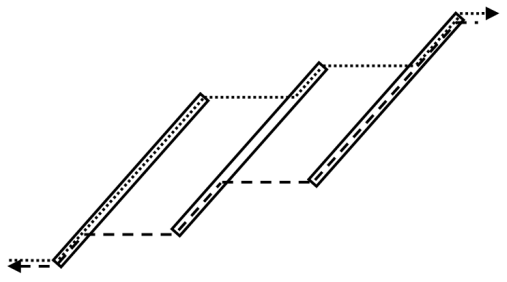

Fig. 11. Occlusions bias the length measure. Neither the dotted nor the dashed path can entirely follow the middle segment.

spatial density of the segments. We use a collection of realizations of a random model of thin, straight segments uniformly distributed in a support of $512 \times 512$ pixels (see Fig. 12). The model contains a population of $N \in\{50,100,150, \ldots, 450\}$ segments of random length $l$ drawn from the normal distribution $\mathcal{N}(\mu, \sigma)$ with $L=\mathcal{N}(40,20)$, bounded in the $(5,90)$ interval. The segments have a random (uniformly distributed) orientation and are placed in the support without touching each other.

We observe that the fragmentation in sparsely populated media $(N=50)$ does not occur, as illustrated in Fig. 13, top, and increases with the density of the population (see the overestimated bin 10 in Fig. 13, bottom). Indeed, the fragmentation cannot be completely avoided even by using the reconstruction since it may happen that no marker remains to reconstruct the segment.

To complete the error evaluation w.r.t. the density of the media see the two following Figs. 14 and 15 that confirm that with increasing number of segments $N$ the error increases 


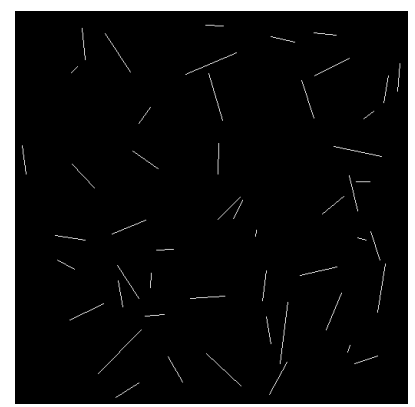

(a) $N=50$

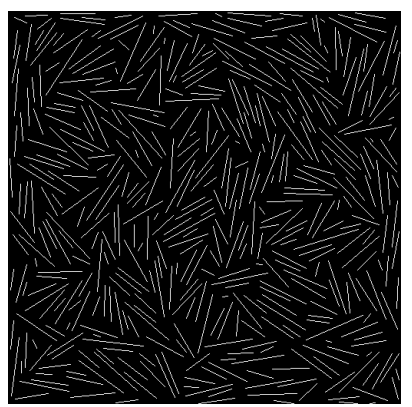

(b) $N=450$

Fig. 12. Two realizations of the random gaussian length distribution model with $N$ segments.
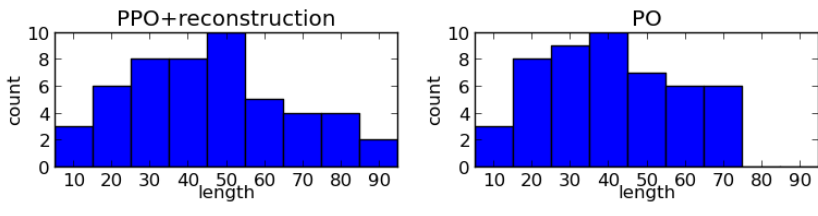

(a) $N=50$ segments
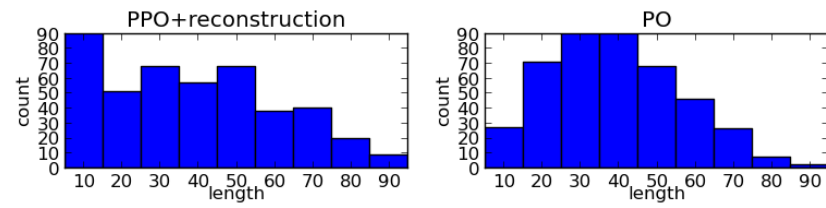

(b) $N=450$ segments
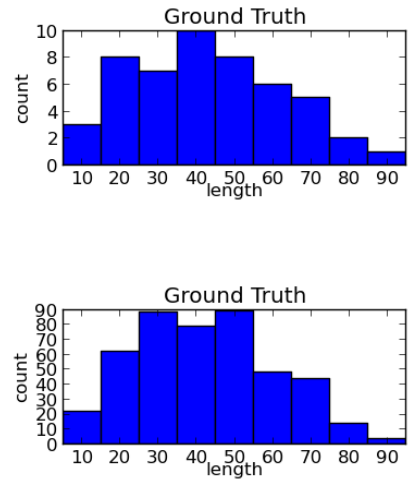

Fig. 13. Length distributions measured with $\mathrm{PPO}+$ reconstruction vs. PO by Talbot and Appleton [20] vs. ground-truth for $N$ segments.

towards stronger underestimation. Both figures are provided for various values of the parameters $k$ and $\beta$. One can observe that the error also increases with increasing parsimony (increasing $k$ ) and decreasing locality (increasing $\beta$ ).

Note that the two length measurement errors, i.e. the overestimation and the one due to occlusions (as in Figs. 10 and 11) are additive. Hence, there are situations where they compensate as e.g. Fig. 14, 50 segments, $k=10$. However, this compensation is illusory since both errors still occur, and their compensation depends of hazardous, random geometrical configurations in the measured structures.

It is interesting to observe however that even for dense populations, as shown in Fig. 12 (right), i.e. a situation deemed unfavorable to a parsimonious approach, PPO parameters can be chosen in such a way that the absolute error is smaller than $10 \%$. The choice of these parameters remains however an open, application dependant, question. For example, the choice of $\epsilon$ is directly linked to the noise level of the image; the higher the noise, the higher $\epsilon$ should be, at the expense of potential unwanted connections between structures that would have been otherwise erased. As it will be seen in the following section, it should be noted that the user has in practice large freedom in the choice of his parameters, as the running time of PPO and PIPO is not impaired by large parameter values.

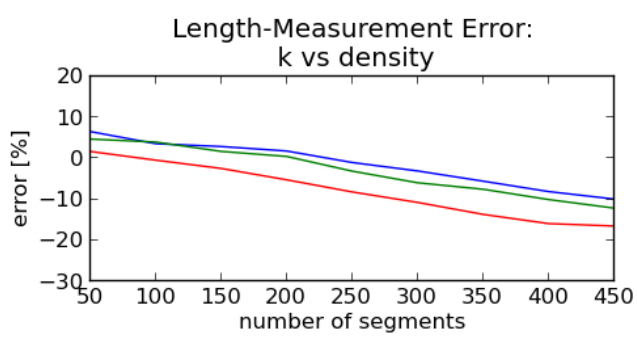

Fig. 14. Relative error w.r.t. to the number of segments and the parsimony parameter: $k=1,5,10$ (blue, green, red).

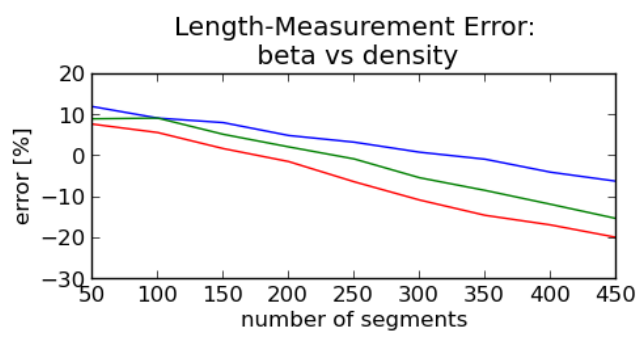

Fig. 15. Relative error w.r.t. the number of segments and the parameter $\beta=$ 1, 20, 50 (blue, green, red).

\section{COMPLEXITY AND TIMINGS}

\section{A. Complexity}

In the original version of $\mathrm{PO}$, the number of paths passing through one pixel is exponential w.r.t. the length $L$. Consequently, if implemented in a naive way, PO have exponential 
complexity. An implementation with logarithmic complexity w.r.t. $L$ has been proposed in [23]. More recently, the complexity of incomplete paths has been decreased to logarithmic w.r.t. $L$ and linear w.r.t. the tolerance $\epsilon$ [20].

Here we analyze the complexity of PPO, split into two parts: path extraction and path filtering.

We have shown above that the collection of paths $\beta M P$ can be extracted in $\mathcal{O}(1)$ per pixel (Sec. V-A), and that an image $f$ can be filtered alongside a path $\pi$ also in $\mathcal{O}(1)$ per sample (Sec. V-B). To complete the analysis of complexity we need to count the number of paths, and evaluate their length.

For a given image $f$, with $D=\operatorname{spt}(f)$ a $W \times H$ rectangle (width $\times$ height), we have $2 W$ vertical and $2 H$ horizontal paths. Vertical paths are $H$ pixels long, and horizontal paths are $W$ pixels long. After dropping multiplicative constants, this yields a linear complexity of $\mathcal{O}(H W)$ when only horizontal and vertical paths are used.

When diagonal paths are used, the complexity slightly increases. We count a total of $4(H+W)$ diagonal paths (for four principal diagonal orientations). The diagonal paths have unequal length, bounded though by $H+W$. This yields a slightly higher complexity of $\mathcal{O}\left((H+W)^{2}\right)$, yet still a linear factor of the support size $\operatorname{card}(D)=H W$.

Therefore, the complexity of PPO and PIPO is proportional to the number of pixels in the images, and independent of the length $L$ and the tolerance $\epsilon$ of incomplete paths, which is an improvement with respect to the state of the art.

\section{B. Timings}

PPO have a low complexity and have been designed to address the timing issues of classical path operators. We compare here the timings of $\mathrm{PO}, \mathrm{RPO}$ and $\mathrm{CPO}$ with the approach used in this paper (PPO). Fig. 16 shows a benchmark for complete and incomplete paths. The gain is huge and we verify that PPO run in constant time with respect to $L$. For complete paths, the computation time is reduced by a factor 75 between PO and PPO, with $k=1$. The gap between incomplete path openings and parsimonious incomplete path openings is even larger. We reduce average time by a factor 3100 with $k=10$.

In another experiment, we benchmark PPO against parameters $\beta, \epsilon$ and $k$, with the same image of size $768 \times 576$. We check in Fig. 17 that the timings are independent of $\beta$ (except for $\beta=1$, which does not use the weighted distance map $\lambda$ ). Regarding the gap tolerance $\epsilon$, an overhead is introduced for $\epsilon>1$ by the closing step. With the parsimony parameter $k$, timings decrease with $\frac{1}{k}$ as expected from the theoretical complexity.

The last benchmark, Fig. 18, exhibits the execution time against the image size. The timings confirm the linear complexity of the algorithm.

\section{CONCLUSIONS}

This paper presents a new family of parsimonious operators for image processing, based on paths. In comparison with

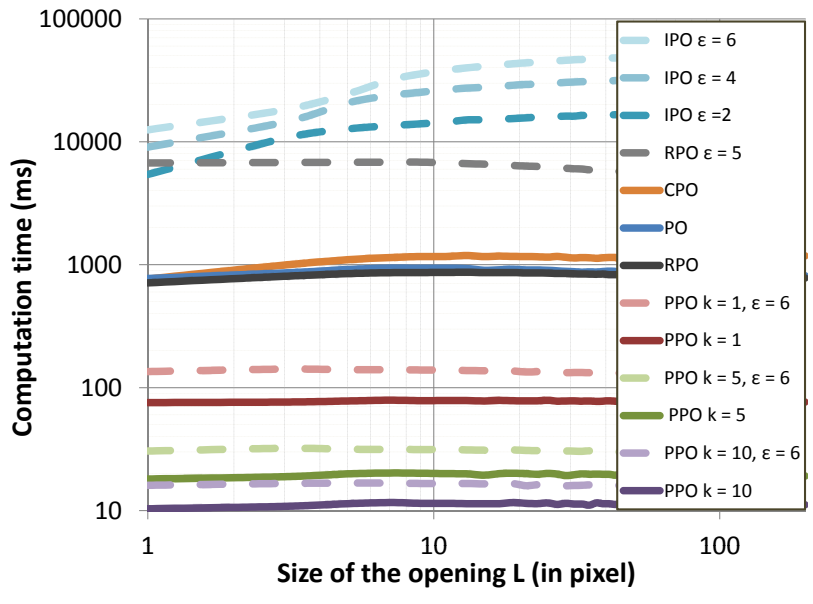

Fig. 16. Benchmark between path openings and parsimonious path openings $(\beta=1)$ for complete and incomplete paths. The timings are plotted in ms w. $\mathrm{r}$. t. $L$ (log-log scale). The input image size is $768 \times 576$ pixels. The benchmarks were made with one thread of a laptop computer (Intel Core i7-2860QM CPU @ $2.50 \mathrm{GHz}$ ) by taking the average value from 100 realizations.

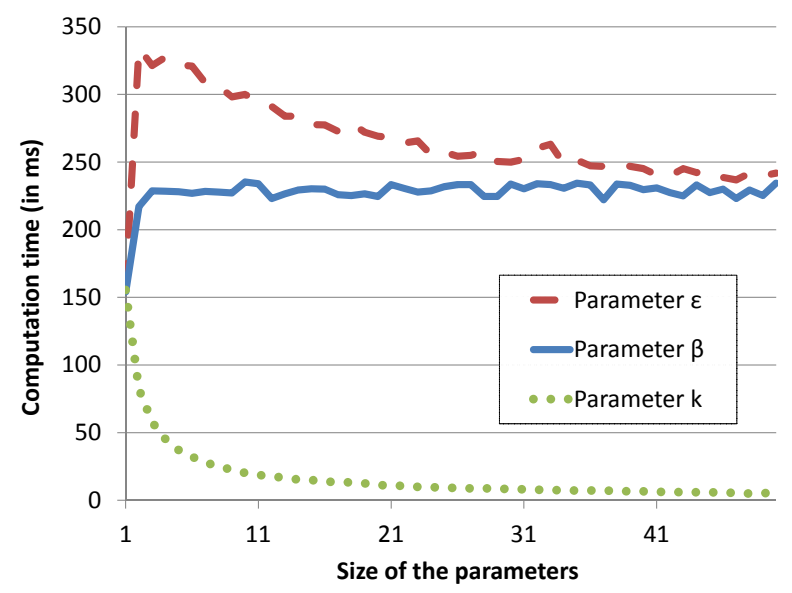

Fig. 17. Influence of the parameters $(\epsilon, \beta$ and $k)$ on the computation time of parsimonious path openings (Intel Core i7-2860QM CPU @ 2.50GHz).

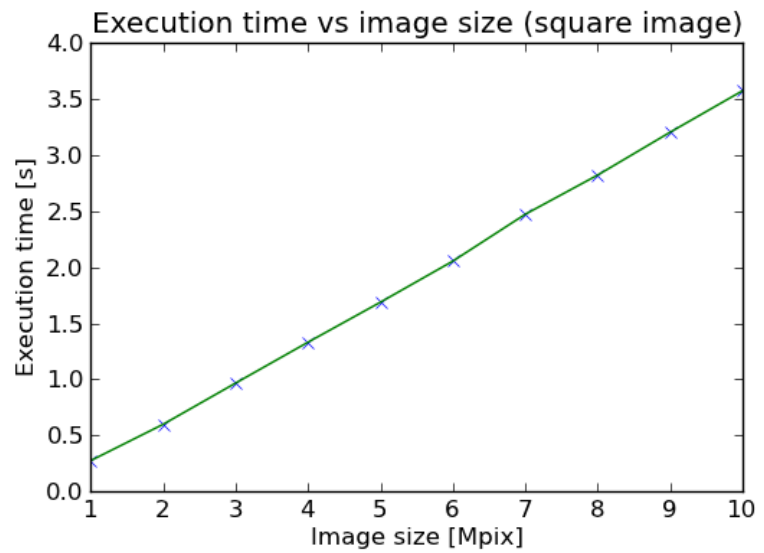

Fig. 18. Execution time of parsimonious path openings $(\beta=1, \epsilon=1, k=1)$ vs image size. 
classical path openings, only a relevant subset of paths is used to retrieve similar information.

The extraction of paths is decoupled from the path filtering, which brings two major advantages: i) a new, general scan strategy allows tuning the search continuously from local to global, which gives the possibility to find a trade-off between accuracy and robustness to noise; ii) it allows defining different operators alongside the same collection of paths, to operate on the same objects. For instance, the combination of a path opening with a closing allows reconnecting discontinued bright structures, thus achieving results similar to those obtained with incomplete path openings, tolerant to missing pixels in paths.

An efficient filtering algorithm [33] is used to compute openings or closings alongside a path. It runs in $\mathcal{O}(1)$ per pixel regardless the size. It decreases the complexity of both path opening and incomplete path opening to a constant. Consequently, the timings are several orders of magnitude lower in comparison with classical (incomplete) path openings, with comparable results. Hence, PPO are usable in highthroughput, industrial applications. Additionally, this filtering algorithm allows : i) using arbitrary data accuracy (integer or floating point), and ii) handling the border effect in two different ways (extending the support with $-\infty_{V}$ or with $\infty_{V}$ ).

We provide a thorough study of the accuracy to show that processing a conveniently chosen subset of paths can provide a result sufficient for certain applications. Such a parsimonious approach has allowed us to bridge the gap between an interesting methodological tool (path openings) and a practical, computation intensive, real-world application.

This work opens different research perspectives.

Parsimonious path operators can be extended to 3D images. In fact, a first series of tests shows that starting from the 3D image borders, and using a 3D graph, gives interesting results. However, parameter $\beta$ has to be chosen with care, since the size of blind regions tends to be larger on 3D images. On the other hand, the fragmentation due to occlusions (cf. Fig. 11) is smaller in $3 \mathrm{D}$.

The proposed strategy only uses paths seeded at the image border. Other starting points could be considered, for example with a random placement, as in the geodesic voting approach [3]. This could help alleviate the blind region problem that may occur sometimes in images with a lot of branching features. Other morphological parsimonious image representations can be also considered, based for example on image extrema, or image ridges and valleys.

Finally, instead of closing-opening, one can also use the rank-max opening to filter the paths in Eq. 10. This will make the PPO behave more like IPO rather than the RPO.

\section{ACKNOWLEDGMENTS}

This work was made possible thanks to the support of the "Pôle ASTech" and the "Pôle Nucléaire de Bourgogne", and has been financed by the French "Département de Seine et Marne"

The authors are grateful to Hugues Talbot (A2SI ESIEE, IGM) and François Cokelaer (IFP) for providing the codes of their respective path-based algorithms.
The authors also wish to thank the anonymous reviewers for their comments.

\section{REFERENCES}

[1] C. Sinthanayothin, J. Boyce, H. Cook, and T. Williamson, "Automated localisation of the optic disc, fovea, and retinal blood vessels from digital colour fundus images," British Journal of Ophthalmology, vol. 83, no. 8, pp. 902-910, 1999.

[2] T. Walter and J.-C. Klein, "Segmentation of color fundus images of the human retina: Detection of the optic disc and the vascular tree using morphological techniques," in Medical Data Analysis, ser. Lecture Notes in Computer Science, J. Crespo, V. Maojo, and F. Martin, Eds. Springer Berlin Heidelberg, 2001, vol. 2199, pp. 282-287. [Online] Available: http://dx.doi.org/10.1007/3-540-45497-7 43

[3] Y. Rouchdy and L. Cohen, "Image segmentation by geodesic voting. application to the extraction of tree structures from confocal microscope images," in Pattern Recognition, 2008. ICPR 2008. 19th International Conference on. IEEE, 2008, pp. 1-5.

[4] V. Bismuth, R. Vaillant, H. Talbot, and L. Najman, "Curvilinear structure enhancement with the polygonal path image-application to guide-wire segmentation in x-ray fluoroscopy," in Medical Image Computing and Computer-Assisted Intervention-MICCAI 2012. Springer, 2012, pp. 9-16.

[5] S. Valero, J. Chanussot, J. Benediktsson, H. Talbot, and B. Waske, "Advanced directional mathematical morphology for the detection of the road network in very high resolution remote sensing images," Pattern Recognition Letters, vol. 31, no. 10, pp. 1120-1127, 2010.

[6] V. Morard, E. Decencière, and P. Dokládal, "Geodesic attributes thinnings and thickenings," in Mathematical Morphology and Its Applications to Image and Signal Processing, Lecture Notes in Computer Science, vol. 6671. Springer, 2011, pp. 200-211.

[7] V. Morard, E. Decencière, and P. Dokládal, "Region growing structuring elements and new operators based on their shape," in 13th IASTED International Conference on Signal and Image Processing (SIP), Proc. of, vol. 759, 2011, pp. 1-8.

[8] J. D. Kurdy B., "Directional mathematical morphology operations," in In 5th European Congress For Stereology, Acta Stereologica, vol. 8/2, 1989.

[9] P. Soille and H. Talbot, "Directional morphological filtering," Pattern Analysis and Machine Intelligence, IEEE Transactions on, vol. 23, no. 11, pp. 1313-1329, 2001.

[10] T. Koller, G. Gerig, G. Szekely, and D. Dettwiler, "Multiscale detection of curvilinear structures in 2-D and 3-D image data," in Computer Vision. Proceedings of the Fifth International Conference on. IEEE, 1995, pp. 864-869.

[11] O. Tankyevych, H. Talbot, and P. Dokládal, "Curvilinear morpho-hessian filter," in 5th IEEE International Symposium on Biomedical Imaging (ISBI), 2008, pp. 1011-1014.

[12] L. Vincent, "Morphological area openings and closings for grey-scale images," in Proceedings of NATO Shape in Picture Workshop. Driebergen, The Netherlands, Springer-Verlag, 1994, pp. 197-208.

[13] E. Breen and R. Jones, "Attribute openings, thinnings, and granulometries," Computer Vision and Image Understanding, vol. 64, pp. 377-389, Nov. 1996.

[14] M. Wilkinson and M. Westenberg, "Shape preserving filament enhancement filtering," in Medical Image Computing and Computer-Assisted Intervention-MICCAI 2001, vol. 2208, 2001, pp. 770-777.

[15] E. Urbach and M. Wilkinson, "Shape-only granulometries and grey-scale shape filters," in Proc. Int. Symp. Math. Morphology (ISMM), vol. 2002, 2002, pp. 305-314.

[16] V. Morard, E. Decencière, and P. Dokládal, "Efficient geodesic attribute thinnings based on the barycentric diameter," Journal of Mathematical Imaging and Vision, vol. 46, no. 1, pp. 128-142, 2013.

[17] M. Buckley and H. Talbot, "Flexible linear openings and closings," Computational Imaging and Vision, vol. 18, pp. 109-118, 2000.

[18] H. Heijmans, M. Buckley, and H. Talbot, "Path openings and closings," Probability, Networks and Algorithms, no. E 0403, pp. 1-21, 2004.

[19] — - "Path openings and closings," Journal of Mathematical Imaging and Vision, vol. 22, no. 2, pp. 107-119, 2005

[20] H. Talbot and B. Appleton, "Efficient complete and incomplete path openings and closings," Image and Vision Computing, vol. 25, no. 4, pp. 416-425, 2007.

[21] F. Cokelaer, H. Talbot, and J. Chanussot, "Efficient Robust dDimensional Path Operators," Selected Topics in Signal Processing, IEEE journal of, vol. 6, no. 7, November 2012. 
[22] J. Serra, Image analysis and mathematical morphology. Academic Press, London, 1982, vol. 1.

[23] B. Appleton and H. Talbot, "Efficient path openings and closings," in Mathematical Morphology: 40 Years On, Computational Imaging and Vision, vol. 30. Springer Netherlands, 2005, pp. 33-42.

[24] C. Luengo Hendriks, "Constrained and dimensionality-independent path openings," Image Processing, IEEE Transactions on, vol. 19, no. 6, pp. 1587-1595, 2010.

[25] R. Bellman, "Dynamic programming," Science, vol. 153, no. 3731, pp. 34-37, 1966.

[26] G. Gallo and S. Pallottino, "Shortest path algorithms," Annals of Operations Research, vol. 13, no. 1, pp. 1-79, 1988.

[27] M. Buckley and J. Yang, "Regularised shortest-path extraction," Pattern Recognition Letters, vol. 18, no. 7, pp. 621-629, 1997.

[28] L. Vincent, "Minimal path algorithms for the robust detection of linear features in gray images," Computational Imaging and Vision, vol. 12, pp. 331-338, 1998.

[29] G. Matheron, Random sets and integral geometry. New York: Wiley, 1974.

[30] M. Schmitt, "Des algorithmes morphologiques à l'intelligence artificielle," Ph.D. dissertation, Ecole Nationale Supérieure des Mines de Paris, 1989.

[31] L. Vincent and D. Jeulin, "Minimal paths and crack propagation simulations," Acta Stereologica, vol. 8, no. 2, pp. 487-494, 1989.

[32] M. Van Droogenbroeck and M. Buckley, "Morphological erosions and openings: fast algorithms based on anchors," Journal of Mathematical Imaging and Vision, vol. 22, no. 2, pp. 121-142, 2005.

[33] V. Morard, P. Dokládal, and E. Decencière, "Linear openings in arbitrary orientation in O(1) per pixel," in Acoustics, Speech and Signal Processing, IEEE International Conference on. IEEE, 2011, pp. 1457-1460.

[34] V. Morard, P. Dokládal, and E. Decencière, "One-dimensional openings, granulometries and component trees in O(1) per pixel," Selected Topics in Signal Processing, IEEE journal of, pp. 1-10, 2011.

[35] P. Karas, V. Morard, J. Bartovský, T. Grandpierre, E. Dokládalová, P. Matula, and P. Dokládal, "GPU Implementation of Linear Morphological Openings with Arbitrary Angle," Journal of Real-Time Image Processing, pp. 1-15, 2012, DOI : 10.1007/s11554-012-0248-7.

[36] L. Vincent, "Morphological grayscale reconstruction in image analysis: Applications and efficient algorithms," Image Processing, IEEE Transactions on, vol. 2, no. 2, pp. 176-201, 1993.

[37] K. Robinson and P. Whelan, "Efficient morphological reconstruction: a downhill filter,' Pattern Recognition Letters, vol. 25, no. 15, pp. 1759 1767, 2004.

[38] G. Matheron, Eléments pour une Thèorie des Milieux Poreux. Masson, 1967.

[39] S.-H. Cha, "Comprehensive Survey on Distance/Similarity Measures between Probability Density Functions," Mathematical Models and Methods in Applied Sciences, Intl. journal of, vol. 1, no. 4, pp. 300-307, 2007.

[40] P. Salembier and J. Serra, "Flat zones filtering, connected operators and filters by reconstruction," IEEE Transactions on Image Processing, vol. 3, no. 8, pp. 1153-1160, 1995.

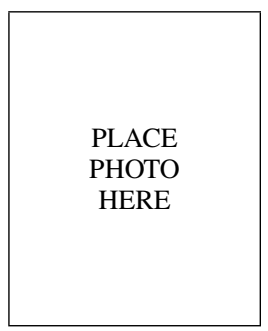

Petr Dokládal Biography text here.

Etienne Decencière Biography text here.

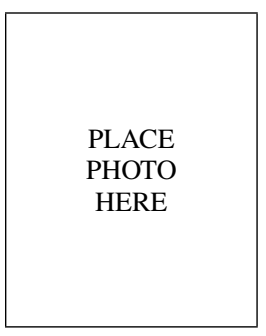

Vincent Morard Biography text here.

PLACE

PHOTO

HERE 